\title{
Segmentation of Q-Ball Images Using Statistical Surface Evolution
}

\author{
Maxime Descoteaux and Rachid Deriche \\ Odyssée Project Team, INRIA/ENS/ENPC, INRIA Sophia Antipolis, France
}

\begin{abstract}
In this article, we develop a new method to segment Q-Ball imaging (QBI) data. We first estimate the orientation distribution function (ODF) using a fast and robust spherical harmonic (SH) method. Then, we use a region-based statistical surface evolution on this image of ODFs to efficiently find coherent white matter fiber bundles. We show that our method is appropriate to propagate through regions of fiber crossings and we show that our results outperform state-of-the-art diffusion tensor (DT) imaging segmentation methods, inherently limited by the DT model. Results obtained on synthetic data, on a biological phantom, on real datasets and on all 13 subjects of a public QBI database show that our method is reproducible, automatic and brings a strong added value to diffusion MRI segmentation.
\end{abstract}

\section{Introduction}

We would like to segment white matter fiber bundles in which diffusion properties are similar and ultimately compare their features to those in other ROI in the same subject or on multiple subjects. Existing DTI-based segmentation techniques $[1,2,3,4,5]$ are inherently limited by the DT model and most often blocked in regions of fiber crossings where DTs are oblate and isotropic. This is why recent high angular resolution diffusion imaging (HARDI) techniques such as QBI [6] have been proposed to aid the inference of crossing, branching or kissing fibers. New methods have thus started to appear to segment bundles in fields of ODFs $[4,7]$. In [4], the ODF map is reconstructed according the time consuming diffusion spectrum imaging (DSI) scheme and the segmentation problem is developed using a level set approach in a non-Euclidean 5-dimensional (5D) position-orientation space. This extension from $3 \mathrm{D}$ to $5 \mathrm{D}$ space leads to work with huge $5 \mathrm{D}$ matrices and there are important problems with data handling and storage. In [7], the main contribution is to model the ODF with a mixture of von Mishes-Fisher distributions and use its associated metric in a hidden Markov measure field segmentation scheme. Thus, both the ODF modeling and segmentation technique are different from our proposed method.

In this paper, we answer the following three questions: 1) How can the segmentation problem be formulated and solved efficiently on a field of diffusion ODFs? 2) What is gained by the ODF with respect to the DT? 3) Is it possible to validate the segmentation results and make the segmentation automatic? To

N. Ayache, S. Ourselin, A. Maeder (Eds.): MICCAI 2007, Part II, LNCS 4792, pp. 769-776, 2007.

(C) Springer-Verlag Berlin Heidelberg 2007 
do so, we propose an efficient region-based level set approach using a regularized and robust spherical harmonics $(\mathrm{SH})$ representation of the ODF [8]. We first show that a better local modeling of fiber crossings improves segmentation results globally. Then, we show that our ODF segmentation is more accurate than the state-of-the-art DTI segmentation [5] in regions of complex fiber configurations from synthetic data, from a biological phantom and from real data. Finally, we show that our Q-ball segmentation is reproducible by segmenting the corpus callosum (CC) of the 13 subjects of a public QBI database [9] automatically.

\section{ODF Estimation from QBI}

QBI [6] reconstructs the diffusion ODF directly from the $N$ HARDI measurements on a single sphere by the Funk-Radon transform (FRT). The ODF is intuitive because it has its maximum(a) aligned with the underlying population of fiber(s). However, computing statistics on a large number of discrete ODF values on the sphere is computationally heavy and infeasible to integrate into a segmentation algorithm of the whole brain. A more compact representation of the ODF is thus needed. $[8,10,11]$ proposed a simple analytic spherical harmonic $(\mathrm{SH})$ reconstruction of the ODF. Letting $Y_{\ell}^{m}$ denote the $\mathrm{SH}$ of order $\ell$ and degree $m(m=-\ell, \ldots, \ell)$ in the standard basis and $Y_{j}\left(j(\ell, m)=\left(\ell^{2}+\ell+2\right) / 2+m\right)$ be the $\mathrm{SH}$ in the modified real and symmetric basis, the final ODF is

$$
\Psi(\theta, \phi)=\sum_{j=1}^{L} \underbrace{2 \pi P_{\ell(j)}(0) c_{j}}_{f_{j}} Y_{j}(\theta, \phi),
$$

where $L=(\ell+1)(\ell+2) / 2, c_{j}$ are the SH coefficients describing the input HARDI signal, $P_{\ell(j)}$ is a Legendre polynomial of order $\ell(j)^{1}$ and $f_{j}$ the coefficients describing the ODF $\Psi$. Here, we use our solution [8] with a Laplace-Beltrami regularization of the $\mathrm{SH}$ coefficients $c_{j}$ to obtain a more robust ODF estimation.

\section{Statistical Surface Evolution}

We want to find a global coherence in the Q-ball field of ODFs. We denote the image of ODFs by $\mathcal{F}: \Omega \mapsto \Re^{L}$ so that for all $\mathbf{x} \in \Omega, \mathcal{F}(\mathbf{x})$ is an ODF of order $\ell$ represented by a vector of $L$ real SH coefficients, $\mathcal{F}(\mathbf{x}):=\left\{f_{1}, \ldots, f_{L}\right\} \in \Re^{L}$. Now, the question is what is a good metric to compare ODFs?

Distances between ODFs. We want to capture similarities and dissimilarities between two ODFs, i.e two spherical functions $\Psi, \Psi^{\prime} \in \mathbf{S}^{2}$ that can be represented by real SH vectors $f, f^{\prime} \in \Re^{L}$, as shown in the previous section. Since the ODFs come from real physical diffusion measurements they are bounded and form an

${ }^{1} \ell(j)$ is the order associated with the $j^{\text {th }}$ element of the SH basis, i.e. for $j=$ $1,2,3,4,5,6,7, \ldots \quad \ell(j)=0,2,2,2,2,2,4, \ldots$ 
open subset of the space of real-valued $\mathcal{L}^{2}$ spherical functions with an inner product $\langle$,$\rangle defined as$

$$
\left\langle\Psi, \Psi^{\prime}\right\rangle=\int_{\sigma \in \mathbf{S}^{2}} \Psi(\theta, \phi) \cdot \Psi(\theta, \phi)^{\prime} d \sigma=\int_{\sigma \in \mathbf{S}^{2}}\left(\sum_{i=1}^{L} f_{i} Y_{i}(\theta, \phi) \sum_{j=1}^{L} f_{j}^{\prime} Y_{j}(\theta, \phi)\right) d \sigma
$$

Because of the orthonormality of the $\mathrm{SH}$ basis, the cross terms cancel and the expression is simply $\left\langle\Psi, \Psi^{\prime}\right\rangle=\sum_{j=1}^{L} f_{j} \cdot f_{j}^{\prime}$. Therefore, the induced $\mathcal{L}^{2}$ norm giving the distance metric between two ODFs is simply $\left\|\Psi-\Psi^{\prime}\right\|=\sqrt{\sum_{j=1}^{L}\left(f_{j}-f_{j}^{\prime}\right)^{2}}$. The Euclidean distance was also used successfully for DTI segmentation in [5] even though more appropriate metrics exist such as the J-Divergence [3,5] and Riemannian geodesic distances [5]. Similarly, one can think of choosing another metric to compare ODFs. For instance, since the ODF can be viewed as a probability distribution function (pdf) of fiber orientations, one can use the KullbackLeibler distance between two pdfs, as done in [6]. However, in that case the problem quickly blows up computationally because one needs to use all $N$ discrete data on the sphere instead of the $L$ SH coefficients $(L<<N) .^{2}$

Segmentation by Surface Evolution. Inspired by general works on image segmentation [12] , we search for the optimal partition $S$ in two regions $S_{1}$ and $S_{2}$ of the image $\Omega$. We maximize the a posteriori frame partition probability $p(S \mid \mathcal{F})$ of obtaining the desired segmentation for the observed image of ODFs $\mathcal{F}$. The major difference in our approach is that we use order-4 ODFs, with $L=15$ real coefficients whereas in [5] DTs represented by $6 \mathrm{D}$ vectors ${ }^{3}$ are used as input to the region-based segmentation.

We use the level set framework to represent the optimal partition $S$ as the zero-crossing of the level set function $\phi$. Hence, using Bayes rule, the optimal partition is obtained by maximizing $p(\phi \mid \mathcal{F}) \propto p(\mathcal{F} \mid \phi) p(\phi)$. At this point, the main assumption is that probability distributions $p_{1}$ and $p_{2}$ of $\mathrm{SH}$ coefficients in regions $S_{1}$ and $S_{2}$ are Gaussians. ${ }^{4}$ Hence, we consider a parametric model with a L-dimensional Gaussian. Letting $\overline{\mathcal{F}}_{r} \in \Re^{L}$ be the mean SH ODF vector and $\Lambda_{r}$ be the $\mathrm{L} \times \mathrm{L}$ covariance matrix of the ODF vectors in region $r=1,2$, the likelihood of the ODF $\mathcal{F}(\mathbf{x})$ to be part of region $r$ is defined as

$$
p_{r}\left(\mathcal{F}(\mathbf{x}) \mid \overline{\mathcal{F}}_{r}, \Lambda_{r}\right)=\frac{1}{(2 \pi)^{3}\left|\Lambda_{r}\right|^{1 / 2}} \exp \left(-\frac{1}{2}\left(\mathcal{F}(\mathbf{x})-\overline{\mathcal{F}}_{r}\right) \Lambda_{r}^{-1}\left(\mathcal{F}(\mathbf{x})-\overline{\mathcal{F}}_{r}\right)\right),
$$

The optimal segmentation is then obtained by maximizing $p(\mathcal{F} \mid \phi) p(\phi)$ or by minimizing of the negative logarithms. Hence, the final energy minimization is

$$
E\left(\phi, p_{1}, p_{2}\right)=-\int_{\Omega} \log p_{1}(\mathcal{F}(\mathbf{x})) d \mathbf{x}-\int_{\Omega} \log p_{2}(\mathcal{F}(\mathbf{x})) d \mathbf{x}+\nu \int_{\Omega} \delta(\phi)|\nabla \phi| d \mathbf{x},
$$

${ }^{2}$ For example, one needs to process $N=200$ values instead of $L=15 \mathrm{SH}$ coefficients.

3 The order-2 SH estimation of the ODF has six coefficients and is related to the DT.

The Euclidean DTI segmentation [5] is thus a special case of the ODF segmentation.

${ }^{4}$ This is in fact a reasonable assumption because we observed "bell-shaped" histograms of each of the $\mathrm{L}$ coefficients of the ODFs in the $\mathrm{CC}$ of our real data. 
where the first two terms are the region-based terms and the last term allows to impose a smoothness constraint $\nu$ on the evolving surface. The Euler-Lagrange equations can then be computed and discretized as in [5] to derive the implicit surface evolution of $\phi$. The most coherent partition is thus obtained in an efficient and simple level set implementation.

\section{Q-Ball Data Generation and Acquisitions}

Synthetic Data We generate synthetic Q-ball data using the multi-tensor model [6], $S\left(\mathbf{u}_{i}\right)=\sum_{k=1}^{n} \frac{1}{n} \exp \left(-b \mathbf{u}_{i}^{\mathrm{T}} \mathbf{D}_{\mathbf{k}}(\theta) \mathbf{u}_{i}\right)+$ noise, for $N$ encoding directions $i \in\{1, \ldots, N\}$. We use $N=81$ from a $3^{r d}$ order tessellation of the icosahedron, $b=3000 \mathrm{~s} / \mathrm{mm}^{2}, n=1$ or 2 and $\mathbf{D}_{\mathbf{k}}(\theta)$ the diffusion tensor with standard eigenvalues $[300,300,1700] \times 10^{-6} \mathrm{~mm}^{2} / \mathrm{s}$ oriented in direction $\theta[6,8]$. The noise is generated with a complex Gaussian noise with a standard deviation of $1 / 35$, producing a signal with SNR 35. We generate two synthetic data example, one with a 2-fiber $90^{\circ}$ crossing (Fig. 1) and the other with a 2-fiber branching configuration (Fig. 2). DTs and ODFs are visualized as spherical functions colored according to the Fractional Anisotropy (FA), with colormap going from red to blue for anisotropic to isotropic profiles.

Biological Phantom Data. We obtained the biological phantom from [13]. It was created from two excised rat spinal cords embedded in $2 \%$ agar. The acquisition was done on $1.5 \mathrm{~T}$ scanner using 90 encoding directions, with $b=$ $3000 \mathrm{~s} / \mathrm{mm}^{2}, \mathrm{TR}=6.4 \mathrm{~s}, \mathrm{TE}=110 \mathrm{~ms}, 2.8 \mathrm{~mm}$ isotropic voxels and four signal averages per direction. We compare the DT Euclidean and Riemannian [5] and ODF surface evolutions on this dataset.

Human Brain Data. First, we use a human brain dataset acquired on $3 \mathrm{~T}$ scanner [14] with 60 encoding directions, $b=1000 \mathrm{~s} / \mathrm{mm}^{2}, 72$ slices with $1.7 \mathrm{~mm}$ thickness, twenty one $b=0 \mathrm{~s} / \mathrm{mm}^{2}$ images, $128 \times 128$ image matrix, TE $=100$ $\mathrm{ms}, \mathrm{TR}=12 \mathrm{~s}$. We compare the segmentations of the DT Euclidean and Riemannian [5] and ODF surface evolutions on two well-known fiber bundles; the corpus callosum (CC) and cortico spinal tract (CST).

Then, we test our ODF segmentation on the public NMR database [9]. The 13 datasets were acquired on a $1.5 \mathrm{~T}$ scanner with 200 encoding directions, $b=$ $3000 \mathrm{~s} / \mathrm{mm}^{2}, 60$ slices with $2 \mathrm{~mm}$ thickness, twenty five $b=0 \mathrm{~s} / \mathrm{mm}^{2}$ images, $128 \times 128$ image matrix, $\mathrm{TE}=93.2 \mathrm{~ms}, \mathrm{TR}=1.9 \mathrm{~s}$. For each subject, a single voxel in the medial part of the $\mathrm{CC}$ is selected (manually) to initialize the flow.

\section{Segmentation Results and Discussion}

Synthetic Datasets. First, Fig. 1 shows that initialization has a strong influence on the final surface. If the initialization contains strictly anisotropic DTs/ODFs, the final surface is not able to pass through the fiber crossing area, as seen in Fig. 1(a). Similarly, the final surface is trapped in the crossing area when 

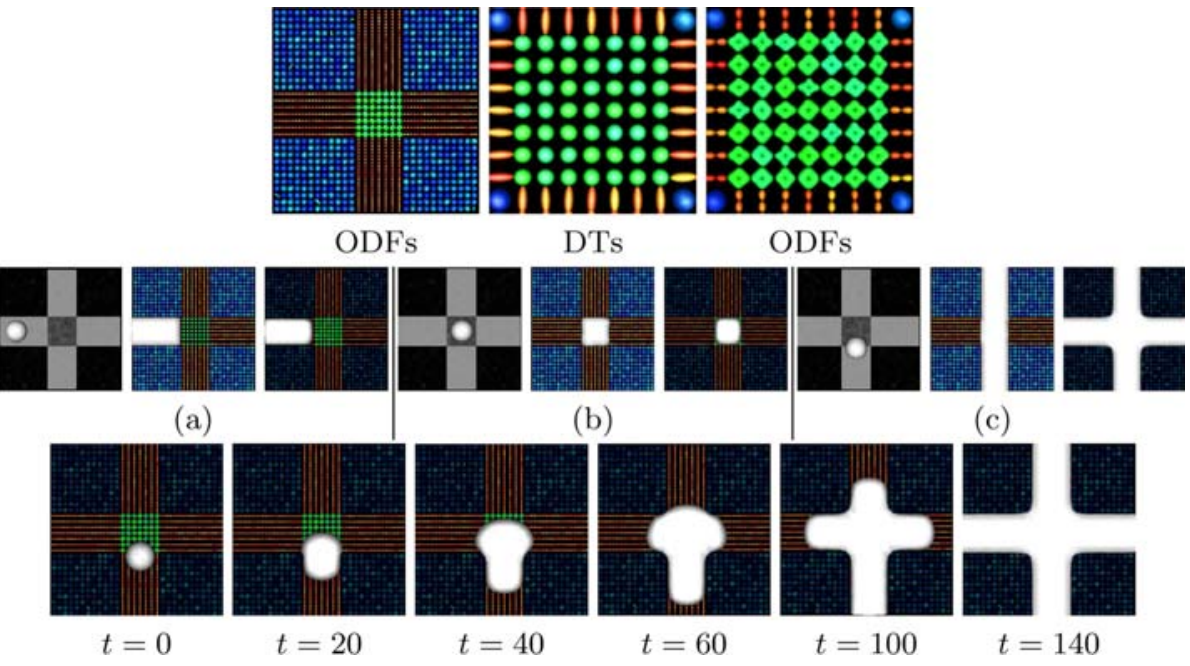

Fig. 1. In the first row, the ODFs and the DTs and ODFs in the $90^{\circ}$ crossing area. In (a-c), from left to right, the initialization used, the DT Riemannian [5] and the ODF flow segmentation. In the last row, the ODF front evolution in time.

initializing strictly in the 2-fiber region (Fig. 1(b)). This is because the statistics of the initial region have a large difference with the rest of the DTs/ODFs and hence, the evolving surface is blocked from connecting to the rest of the structure. However, if the initialization contains a mixture of both single fiber and 2-fiber DTs/ODFs, the DT flow propagates through the crossing region to connect to the similar anisotropic DTs on the other side of the crossing and the second fiber is completely ignored, as seen in Fig. 1(c). The DTs in the crossing are oblate and there is no information on the second orientation. In contrary, there is information about the second orientation in the ODF flow and the surface evolution finds the whole 2-fiber structure as coherent. Fig. 2 shows a more complex branching region. In the DT flow, we see that the surface remains trapped in the regions of the initial seeding for all initializations. In contrary, in the ODF case, when the flow is initialized in the bottom and middle part of the branch, the whole branching structure is recovered because the ODF contains a broader range of orientations in its statistics.

Biological Phantom Dataset. Fig. 3 shows that the DT flow with the Euclidean distance is unable to segment the spinal cords. Whereas in [5] the initialization was placed outside the phantom and the flow converged inwards, here, we initialized inside the structure and we see that the surface leaks outside the cords because many DTs are isotropic in the fibers and there are also isotropic DTs outside the structure with mean diffusivity in a similar range. However, our new ODF flow segments the whole structure quite easily. The segmentation agrees with results published using the DT Riemannian flow [5, Fig.12-13]. 

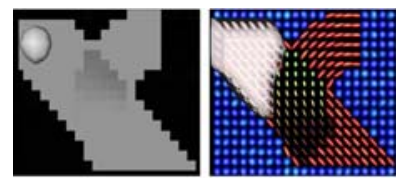

(a)
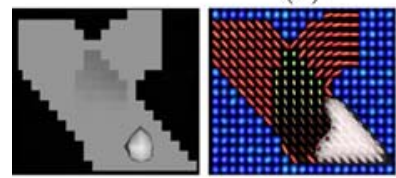

(c)
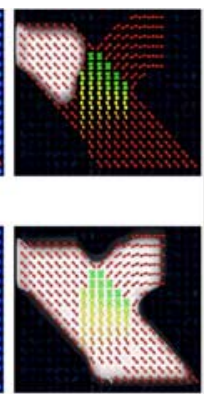

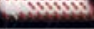
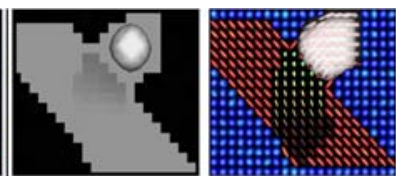

(b)
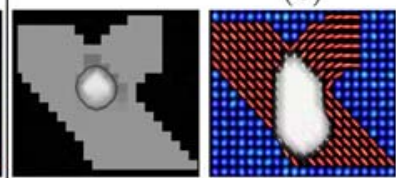

(d)
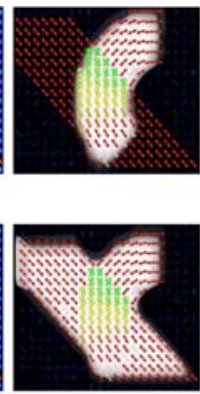

winstivs

Fig. 2. Segmentation on a synthetic branching example. In (a-d), from left to right, the initialization used, the DT Riemannian [5] and the ODF flow segmentation.

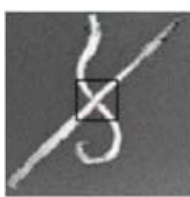

T1

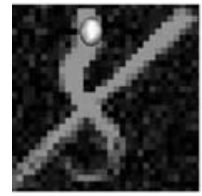

(b) -

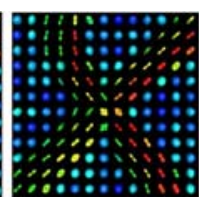

ODFs
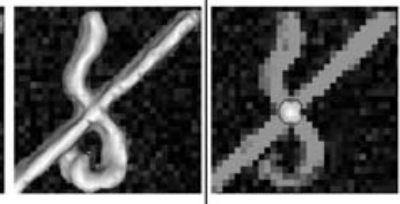

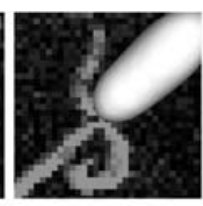

(a)

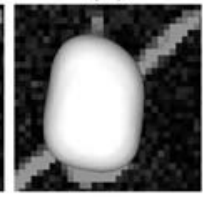

(c)
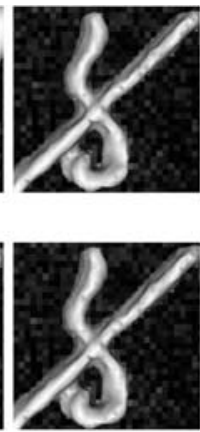

Fig. 3. In (a-c), from left to right, the initialization, the DT Euclidean [5] flow at $t=40$ starting to leak outside the phantom structure and the segmentation of the ODF flow

Human Brain Datasets. Our ODF segmentation on real datasets recovers more structure than other published results on the CC and CST [1,4,5]. Fig. 4 shows that we are able to reproduce results from [5] with the DT-based flows using both the Euclidean and Riemannian distances. In the DT Euclidean flow, we see that the evolving surface stops near complex crossing area where oblate and isotropic DTs (greenish-blue) block the flow. The DT Riemannian is able to connect more voxels than the DT Euclidean by slightly evolving into the crossing area. However, in the CST, the flow is still unable to recover the branching fiber structure projecting to the cortex. The ODF flow recovers that branching structure to the different sulci and also recovers more of the splenium of the CC.

Fig 5 shows that our new ODF surface evolution is reproducible on many subjects from the same set parameters. Convergence depends on the subject but was always obtained automatically for 80 to 120 iterations of the flow, where an iteration takes roughly 0.5 second on a Dell single processor, $3.4 \mathrm{GHz}, 2 \mathrm{~GB}$ RAM machine. We see that for most subjects, we have segmented the full CC with the longer posterior parts of the splenium and the full genu, as in Fig. 4. 

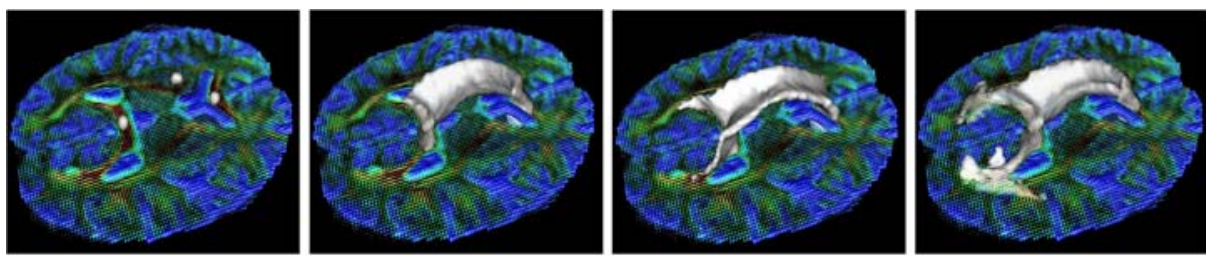

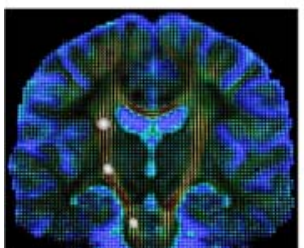

Initialization

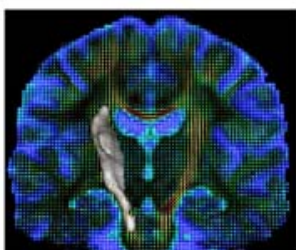

DT Euclidean [5]

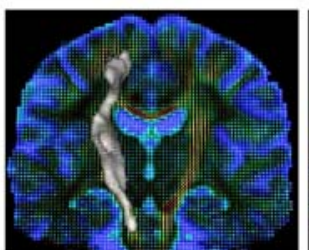

DT Riemannian [5]

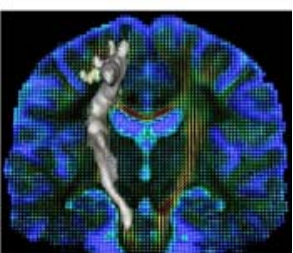

ODF flow

Fig. 4. ODF flow segmentations can propagate through crossing regions and go further than other segmentation methods. DT-based segmentations are overlaid on a slice with DTs and the ODF flow is overlaid on the same slice with the ODFs.
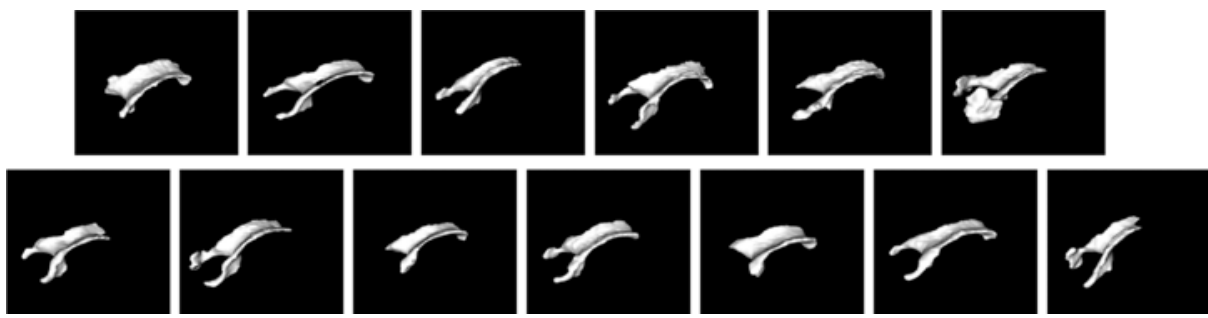

Fig. 5. Automatic segmentation of the corpus callosum using the ODF flow on the 13 subjects of the NMR database [9] from a single seed point in the middle of the CC

Overall all CC structures are similar but there are some differences across CCs. Hence, it is now important to quantify this multi-subject variability.

\section{Conclusion}

We have presented an efficient statistical surface evolution framework for the segmentation of Q-Ball images. The proposed method combines state-of-theart $\mathrm{SH}$ reconstruction of the ODF from QBI and state-of-the-art region-based surface evolution. To answer questions of the introduction: 1) The segmentation problem on ODF images can be formulated efficiently with the level sets evolving to partition similar ODF based on their spherical harmonic representation. 2) The ODF flow is able to deal with complex fiber configurations such as crossing and branching fibers better than DT-based segmentation using the Euclidean and Riemannian distances. 3) It is possible to validate the segmentation results. In particular, we obtained sets of globally coherent ODFs agreeing with 
well-known real data cerebral anatomical structures as well as with synthetic and biological phantom datasets where the ground truth was known. Another important contribution was to show the reproducibility of the surface evolution on real datasets with different $b$-values and also on the 13 subjects from the public NMR database. It is now important to develop a better initialization of the level set front in order to perform a fully automatic segmentation. It is now possible to imagine performing a multi-subject study with segmented fiber bundles to quantify certain diffusion properties and attempt to follow the evolution of white matter diseases.

\section{References}

1. Zhukov, L., Museth, K., Breen, D., Whitakert, R., Barr, A.H.: Level set modeling and segmentation of DT-MRI brain data. J. of Electronic Imaging 12, 125-133 (2003)

2. Feddern, C., Weickert, J., Burgeth, B.: Level-set methods for tensor-valued images. In: Proceedings of the Second IEEE Workshop on Geometric and Level Set Methods in Computer Vision, pp. 65-72. IEEE Computer Society Press, Los Alamitos (2003)

3. Wang, Z., Vemuri, B.C.: DTI segmentation using an information theoretic tensor dissimilarity measure. IEEE Trans. in Medical Imaging 24(10), 1267-1277 (2005)

4. Jonasson, L.: Segmentation of diffusion weighted MRI using the level set framework. PhD thesis, Ecole Polytechnique federale de Lausanne (2006)

5. Lenglet, C., Rousson, M., Deriche, R.: DTI segmentation by statistical surface evolution. IEEE Transactions in Medical Imaging 25(6), 685-700 (2006)

6. Tuch, D.: Q-ball imaging. Magnetic Resonance in Medicine 52(6), 1358-1372 (2004)

7. McGraw, T., Vemuri, B., Yezierski, R., Mareci, T.: Segmentation of high angular resolution diffusion MRI modeled as a field of von Mises-Fisher mixtures. In: Leonardis, A., Bischof, H., Pinz, A. (eds.) ECCV 2006. LNCS, vol. 3953, pp. 463475. Springer, Heidelberg (2006)

8. Descoteaux, M., Angelino, E., Fitzgibbons, S., Deriche, R.: Regularized, fast, and robust analytical Q-ball imaging. Magnetic Resonance in Medicine (to appear)

9. Poupon, C., Poupon, F., Allirol, L., Mangin, J.F.: A database dedicated to anatomo-functional study of human brain connectivity. In: HBM. Twelfth Annual Meeting of the Organization for Human Brain Mapping (2006)

10. Anderson, A.: Measurements of fiber orientation distributions using high angular resolution diffusion imaging. Magnetic Resonance in Medicine 54, 1194-1206 (2005)

11. Hess, C., Mukherjee, P., Han, E., Xu, D., Vigneron, D.: Q-ball reconstruction of multimodal fiber orientations using the spherical harmonic basis. Magnetic Resonance in Medicine 56, 104-117 (2006)

12. Paragios, N., Deriche, R.: Geodesic active regions: a new paradigm to deal with frame partition problems in computer vision. Journal of Visual Communication and Image Representation 13(1/2), 249-268 (2002)

13. Campbell, J., Siddiqi, K., Rymar, V., Sadikot, A., Pike, B.: Flow-based fiber tracking with diffusion tensor Q-ball data: Validation and comparison to principal diffusion direction techniques. NeuroImage 27(4), 725-736 (2005)

14. Anwander, A., Tittgemeyer, M., von Cramon, D.Y., Friederici, A.D., Knosche, T.R.: Connectivity-based parcellation of BROCA's area. Cerebral Cortex 17(4), 816-825 (2007) 